\title{
Radiometric Calibration for a Multispectral Sensor Onboard RISESAT Microsatellite Based on Lunar Observations
}

\author{
Masataka Imai ${ }^{1,2} \oplus$, Junichi Kurihara ${ }^{3, *}\left(\mathbb{0}\right.$, Toru Kouyama ${ }^{1,2} \oplus$, Toshinori Kuwahara ${ }^{4}$, Shinya Fujita ${ }^{4}$, \\ Yuji Sakamoto ${ }^{4}$, Yuji Sato ${ }^{4}$, Sei-Ichi Saitoh ${ }^{5}$, Takafumi Hirata ${ }^{5}$, Hirokazu Yamamoto ${ }^{6}$ and Yukihiro Takahashi ${ }^{3}$ \\ 1 Artificial Intelligence Research Center, National Institute of Advanced Industrial Science and Technology, \\ Tokyo 135-0064, Japan; mstk-a.imai@aist.go.jp (M.I.); t.kouyama@aist.go.jp (T.K.) \\ 2 AIST-UTokyo Advanced Operando-Measurement Technology Open Innovation Laboratory \\ (OPERANDO-OIL), National Institute of Advanced Industrial Science and Technology (AIST), \\ Kashiwa 277-0882, Japan \\ 3 Faculty of Science, Hokkaido University, Sapporo 001-0021, Japan; yukihiro@sci.hokudai.ac.jp \\ 4 Department of Aerospace Engineering, Tohoku University, Sendai 980-8579, Japan; \\ toshinori.kuwahara.b3@tohoku.ac.jp (T.K.); shinya.fujita.a2@tohoku.ac.jp (S.F.); \\ yuji.sakamoto.b4@tohoku.ac.jp (Y.S.); yuji.sato.s4@dc.tohoku.ac.jp (Y.S.) \\ 5 Arctic Research Center, Hokkaido University, Sapporo 001-0021, Japan; ssaitoh@arc.hokudai.ac.jp (S.-I.S.); \\ tahi@arc.hokudai.ac.jp (T.H.) \\ 6 Geological Survey of Japan, National Institute of Advanced Industrial Science and Technology, \\ Ibaraki 305-8567, Japan; hirokazu.yamamoto@aist.go.jp \\ * Correspondence: kurihara@sci.hokudai.ac.jp; Tel.: +81-11-706-9244
}

\section{check for} updates

Citation: Imai, M.; Kurihara, J.; Kouyama, T.; Kuwahara, T.; Fujita, S.; Sakamoto, Y.; Sato, Y.; Saitoh, S.-I.; Hirata, T.; Yamamoto, H.; et al. Radiometric Calibration for a Multispectral Sensor Onboard RISESAT Microsatellite Based on Lunar Observations. Sensors 2021, 21, 2429. https://doi.org/10.3390/ s21072429

Academic Editor: Maorong Ge

Received: 16 February 2021

Accepted: 31 March 2021

Published: 1 April 2021

Publisher's Note: MDPI stays neutral with regard to jurisdictional claims in published maps and institutional affiliations.

Copyright: (c) 2021 by the authors. Licensee MDPI, Basel, Switzerland. This article is an open access article distributed under the terms and conditions of the Creative Commons Attribution (CC BY) license (https:// creativecommons.org/licenses/by/ $4.0 /)$.

\begin{abstract}
Radiometric calibration utilizing the Moon as a reference source is termed as lunar calibration. It is a useful method for evaluating the performance of optical sensors onboard satellites orbiting the Earth. Lunar calibration provides sufficient radiometric calibration opportunities without requiring any special equipment, and is suitable for nano/microsatellites. This study applies lunar calibration to a multispectral sensor, Ocean Observation Camera (OOC), on board a microsatellite named Rapid International Scientific Experiment Satellite. Simulating the brightness of the Moon based on the RObotic Lunar Observatory and SELENE/Spectrum Profiler models, sensitivity degradation was proven to be negligible in any of the four spectral bands of the OOC with the sensor temperature correction. A bluing trend in the OOC's sensor sensitivity was revealed, indicating a shorter observation wavelength shows larger irradiance. Comparing the top-of-atmosphere reflectance of Railroad Valley Playa with the Radiometric Calibration Network dataset revealed that the derived calibration parameter from the lunar calibration was valid for correcting the bluing trend in the visible range. Although the lunar and vicarious calibration parameters for the infrared band were unexpectedly inconsistent, lunar calibration could potentially contribute toward estimating the contaminated background radiance in the Earth observation images.
\end{abstract}

Keywords: remote sensing; Earth observation; nano/microsatellite; lunar calibration

\section{Introduction}

Earth observation by nano/microsatellites has been developing rapidly over the past decade [1]. The principal reason for this growing trend is the advantage of nano/microsatellites over large satellites in providing frequent observations of the Earth's surface by satellite constellations. The PlanetScope constellation having the largest number of satellites in Earth observation with more than 130 nanosatellites in orbit, provides daily imagery of the entire Earth's surface. In contrast, even a virtual constellation of the large Earth observation satellites composed of Landsat-8, Sentinel-2A, and Sentinel-2B provides an average revisit interval of 2.9 days [2]. The unprecedented high temporal resolution of the nanosatellite constellation opens new possibilities for Earth observation for the detection of short-term changes and acquisition of instantaneous information in case of illegal forest logging [3], water quality variations [4], and oil spill accidents [5]. 
However, in terms of radiometric precision, nano/microsatellite sensors still consist of many disadvantages arising predominantly due to size limitations and the use of commercial off-the-shelf components [6]. Significant radiometric inconsistencies exist between different satellites in the PlanetScope constellation [7]. Although several normalization methods have been developed for PlanetScope imagery using satellite images from Landsat8, MODIS (Moderate Resolution Imaging Spectroradiometer), and Sentinel-2 [6-10], the acquisition time difference between the nano and large satellites erodes the advantage of the nanosatellite imagery with higher temporal resolution. In order to ensure radiometric precision, radiometric calibration is necessary for both nano/microsatellite as well as large satellite sensors, and thus, routine calibration and validation needs to be conducted. As nano/microsatellite sensors are relatively sensitive to launch vibration and space radiation environments, the on-orbit radiometric characteristics of the sensor may deviate from the pre-launch radiometric characteristics in both short-and long-time scales. Therefore, periodic, frequent on-orbit calibration for nano/microsatellite is very important to confirm variations in radiometric characteristics.

Onboard calibration hardware, such as solar diffuser panels and calibration lamp sources, generally employed for the on-orbit calibration of large satellite sensors [11], cannot be equipped on nano/microsatellites due to size limitations. Alternatively, vicarious and lunar calibrations can be applied for the on-orbit calibration of nano/microsatellite sensors. In case of vicarious calibration, sites such as dry lakes and deserts on the surface of the Earth, with known or measured ground-based reflectance values are chosen as calibration targets. The uncertainty of vicarious calibration depends on atmospheric measurements and cloud conditions at the site [12]. Inter-satellite observations with similar channels over Simultaneous Nadir Overpass (SNO) can also be used as a tool for calibration and relative stability monitoring. The uncertainty of cross-calibration with SNO approach depends mainly on radiometric precision of the reference satellite sensor. Lunar calibration uses the surface of the Moon as a calibration target, for which the reflectance was modeled from ground-based measurements and previous lunar missions. Although the reflectance of the Moon surface has the advantage of being highly stable (less than $1 \%$ variation over 1 billion years [13]) with no atmospheric effects, the actual implementation of lunar calibration is a challenging task for nano/microsatellites, which are controlled by limited attitude accuracy and power availability. While PlanetScope recently implemented a rigorous radiometric correction including lunar calibration $[14,15]$, there are very few studies on the lunar calibration of nano/microsatellite sensors [16,17].

This paper demonstrates the results of lunar calibration applied to Ocean Observation Camera (OOC) on the Rapid International Scientific Experiment Satellite (RISESAT) microsatellite to establish a convenient and reliable radiometric calibration method for nano/microsatellite sensors. The objective of the OOC mission is to conduct ocean color remote sensing, which requires a relatively high radiometric precision among various fields of Earth observation. Compared with previous studies on the lunar calibration of nano/microsatellite sensors, the new approaches implemented in this study were (1) to conduct periodic and frequent Moon observations by the OOC for more than a year; (2) to use two different Moon models for radiometric calibration; and (3) to apply the sensor temperature of the OOC to the radiometric correction.

\section{Materials and Methods}

\subsection{Satellite Sensor and Imagery}

The OOC is one of the scientific instruments onboard the RISESAT microsatellite, which was launched on 18 January 2019, into a sun-synchronous orbit at an altitude of $500 \mathrm{~km}$ (Figure 1). The satellite bus system was developed by Tohoku University, and the scientific payload instruments were selected internationally from a wide variety of missions in Earth observation and space science $[18,19]$. The OOC was developed predominantly by Hokkaido University with funding and technical support from the National Taiwan Ocean University, PASCO Corporation, and Tohoku University. The objective of the OOC is to 
conduct ocean color remote sensing, which is focused on the colored dissolved organic matter (CDOM) in coastal waters. The OOC is a two-dimensional multispectral imager, with four cameras (OOC-1/2/3/4) corresponding to different spectral bands in the visible and near-infrared regions (Table 1). A bandwidth of approximately $10 \mathrm{~nm}$ (Figure 2) is typically required for ocean color sensors [20]. As an ocean color sensor, the OOC has a unique spectral band of OOC- 1 at $405 \mathrm{~nm}$ dedicated for CDOM estimation. The spectral bands of OOC-2 and 3 are at $490 \mathrm{~nm}$ and $555 \mathrm{~nm}$, respectively, and are conventionally applied for the estimation of chlorophyll-a concentration. In addition, the spectral band of OOC-4 at $869 \mathrm{~nm}$ is used for atmospheric correction. Each of the four cameras are composed of an optical bandpass filter (Andover Corporation, NH, USA), a lens with a fixed focal length of $50 \mathrm{~mm}$ (MORITEX Corporation, Saitama, Japan), and a charge-coupled device (CCD) image sensor (Watec Co., Ltd., Yamagata, Japan), which are commercially available at low costs. The four cameras are installed in a single housing with temperature sensor attached to the outer surface. In most large satellites, the sensor temperatures are precisely measured and controlled at each component. Contrarily, owing to the size and power limitations, the OOC contains a single temperature sensor in the housing with no active thermal control being performed.

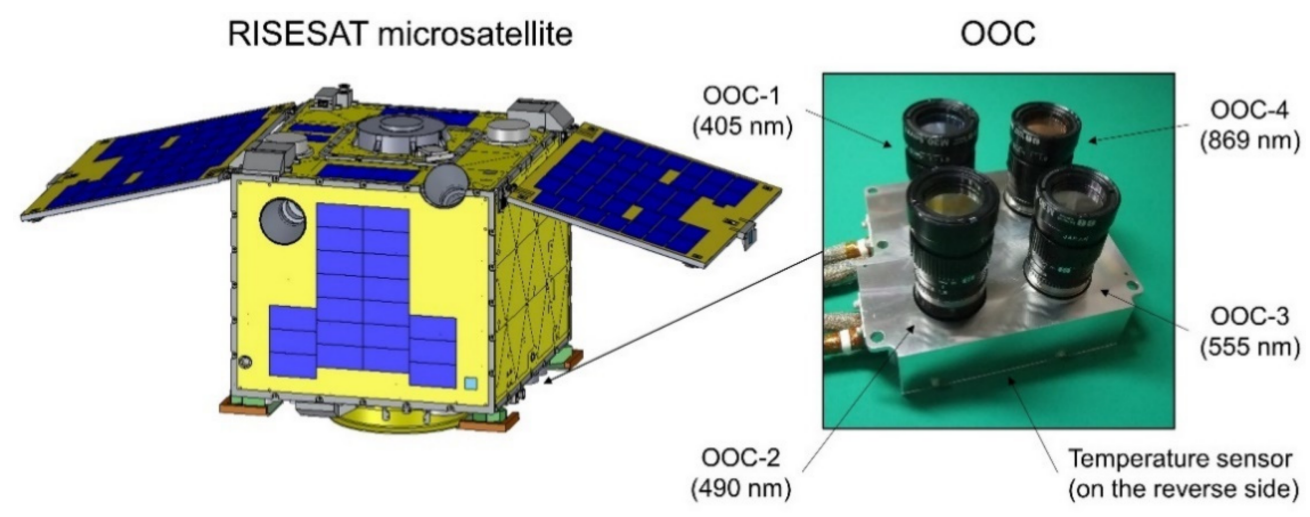

Figure 1. An overview of the RISESAT microsatellite and the Ocean Observation Camera (OOC) with its four cameras each corresponding to different spectral bands.

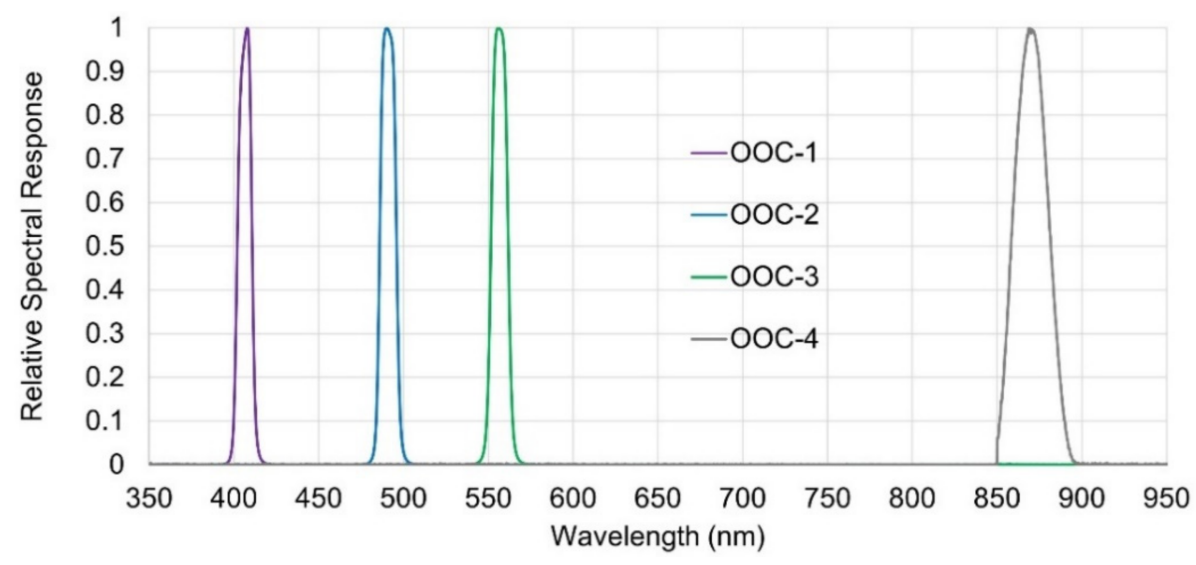

Figure 2. Relative spectral response functions of the OOC. 
Table 1. Specifications of the OOC.

\begin{tabular}{cc}
\hline Size & $388 \times 161 \times 124 \mathrm{~mm}$ \\
Weight & $0.8 \mathrm{~kg}$ \\
Ground Sample Distance & $74 \mathrm{~m}($ at $500 \mathrm{~km}$ alt. $)$ \\
Field of View & $5.6^{\circ} \times 4.2^{\circ}(48 \times 36 \mathrm{~km}$ at $500 \mathrm{~km}$ alt. $)$ \\
\hline & OOC-1: $405 \mathrm{~nm}$ \\
Spectral Bands & OOC-2: $490 \mathrm{~nm}$ \\
& OOC-3: $555 \mathrm{~nm}$ \\
Image Size & OOC- $4: 869 \mathrm{~nm}$ \\
\hline Data Quantization & $659 \times 494$ pixels \\
\end{tabular}

Moon observations by the OOC started on August 16, 2019, after seven months of the initial launch checkout and preliminary observations for the scientific instruments onboard the RISESAT microsatellite. RISESAT was originally designed to track a ground target with an accuracy of $0.1^{\circ}$ and $0.008^{\circ} / \mathrm{s}$ attitude stability. A high-speed attitude control computer and precision ground-tracking algorithms have been developed for this purpose [21-23]. This onboard attitude control system enabled the OOC to look at the Earth's surface along with other astronomical objects including the Moon and other planets in our solar system. Table 2 provides a detail summary of the Moon observations by the OOC. The Moon surface brightness varies largely at a phase angle $|\alpha| \leq 7^{\circ}$, as a strong backscattering or brightness opposition effect occurs at this value [24]. Thus, Moon observations were carried at an absolute phase angle of approximately $10^{\circ}\left( \pm 2.5^{\circ}\right)$ to obtain the maximum brightness, avoiding the backscattering surge. The observations were conducted every month until April 2020 and were repeated every 3-4 months afterward. The OOC acquired the Moon images with the four bands simultaneously, and multiple images were taken at intervals of a few seconds for each observation time.

Table 2. Summary of the OOC's Moon observations with phase angle and the sensor housing temperature listed for individual observation time. The number of obtained Moon images are indicated in the OOC band columns. Negative phase angles indicate a waxing Moon.

\begin{tabular}{|c|c|c|c|c|c|c|}
\hline \multirow{2}{*}{ Observation Time } & \multicolumn{4}{|c|}{ OOC Band } & \multirow{2}{*}{$\begin{array}{c}\text { Phase } \\
\left.\text { Angle ( }{ }^{\circ}\right)\end{array}$} & \multirow{2}{*}{$\begin{array}{c}\text { Sensor } \\
\text { Temperature }\left[{ }^{\circ} \mathrm{C}\right]\end{array}$} \\
\hline & 1 & 2 & 3 & 4 & & \\
\hline 2019-08-16 05:00:52-05:01:08 & 4 & 5 & 5 & 5 & -7.7 & 27.0 \\
\hline $12: 53: 30-12: 54: 30$ & 5 & 5 & 5 & 5 & -11.1 & 29.0 \\
\hline $16: 02: 30-16: 03: 30$ & 5 & 5 & 5 & 5 & -12.5 & 30.0 \\
\hline 2019-09-14 23:56:31-23:57:31 & 5 & 5 & 5 & 5 & 11.0 & $19.9-20.9$ \\
\hline 2019-10-12 22:41:30-22:42:30 & 4 & 4 & 5 & 5 & 11.0 & 19.9 \\
\hline 2019-11-11 16:01:30-16:02:30 & 5 & 4 & 5 & 5 & 10.7 & 24.0 \\
\hline 2019-12-11 12:27:30-12:28:30 & 5 & 5 & 5 & 5 & 8.6 & $21.9-22.9$ \\
\hline $15: 37: 30-15: 38: 30$ & 5 & 5 & 5 & 4 & 7.0 & $24.0-25.0$ \\
\hline 2020-01-09 21:51:30-21:52:30 & 4 & 5 & 5 & 5 & -11.7 & 29.0 \\
\hline 2020-01-10 01:00:30-01:01:30 & 5 & 5 & 5 & 4 & -10.0 & 15.9 \\
\hline 04:09:30 & 0 & 1 & 0 & 1 & -8.5 & 15.9 \\
\hline 2020-02-08 11:55:31-11:56:31 & 5 & 5 & 5 & 5 & -12.0 & 29.0 \\
\hline 15:04:45-15:05:30 & 4 & 4 & 4 & 4 & -10.4 & 26.0 \\
\hline 18:13:30-18:14:30 & 5 & 5 & 5 & 5 & -8.9 & 31.0 \\
\hline 2020-03-09 00:22:30-00:23:30 & 5 & 5 & 5 & 5 & -12.1 & 19.9 \\
\hline 03:31:30-03:32:30 & 4 & 5 & 4 & 5 & -10.5 & 22.9 \\
\hline 06:40:30-06:41:30 & 2 & 5 & 5 & 2 & -9.1 & $22.9-24.0$ \\
\hline 2020-04-07 23:48:30-23:49:30 & 1 & 5 & 1 & 1 & -6.0 & 15.9 \\
\hline 2020-04-08 02:57:30-02:58:30 & 3 & 3 & 4 & 5 & -5.5 & 19.9 \\
\hline $13: 57: 30-13: 58: 00$ & 1 & 2 & 1 & 1 & -7.8 & 16.9 \\
\hline 17:06:30-17:07:30 & 0 & 4 & 2 & 1 & -9.2 & 18.9 \\
\hline 2020-08-03 00:18:30-00:19:30 & 3 & 3 & 3 & 3 & -8.4 & $9.9-10.9$ \\
\hline 2020-11-29 11:48:30-11:49:30 & 3 & 3 & 3 & 3 & -9.6 & 30.0 \\
\hline
\end{tabular}




\subsection{Lunar Radiometric Models}

Owing to the fact that the reflectance of the Moon's surface is extremely stable, many studies have been conducted to utilize the Moon as a photometric standard. The major difficulty of using the Moon as an absolute radiometric standard exists in its strong brightness variations against solar illumination and viewing geometry. The U.S. Geological Survey in Flagstaff established the ground-based RObotic Lunar Observatory (ROLO) and developed a Moon model [25]. The ROLO model can predict the brightness of the Moon (irradiance) with a precision of $\sim 1 \%$ over a wide phase range. Using the ROLO model, inter-satellite calibration between Sea-WiFS and MODIS was demonstrated [26]. Currently the ROLO model and its implementation have been widely used in on-going satellites to detect the sensor degradation, such as Suomi National Polar-orbiting Partnership Visible Infrared Imaging Radiometer Suite, Landsat-8 Operational Land Imager, and so on [27,28].

Recently, another Moon model was developed using spacecraft observation data obtained by the Spectrum Profiler (SP) onboard the SELENE Japanese Moon orbiter [29,30]. The SP model can reproduce the Moon global brightness against any illumination and the viewing condition for any Moon location (including the opposite side of the Moon). This model has the advantage of calibrating an optical sensor with a higher spatial resolution and a field of view (FOV) narrower than the size of a disk. Although the absolute radiometric precision of the SP model $(\sim 10 \%)$ is large compared to that of the ROLO model, the measurement of relative sensor degradation of the order of $0.1 \%$ has been achieved [31].

In this study, both the ROLO and SP models were used to simulate the Moon irradiance received at the satellite position in the Earth orbit at each observation time. The SP model simulates the lunar surface radiance at each grid point specified by the solar incident angle $(i)$, emission angle $(e)$, and phase angle $(\alpha)$ (Figure 3$)$. The photometrically corrected reflectance $r_{\text {corr }}$ is provided within the SP model, where $i=30^{\circ}, e=0^{\circ}$, and $\alpha=30^{\circ}$ is employed as a standard viewing geometry, and the radiance factor $r_{\text {sim }}$ is calculated as

$$
r_{\text {sim }}(\lambda, i, e, \alpha)=r_{\text {corr }}\left(\lambda, 30^{\circ}, 0^{\circ}, 30^{\circ}\right) \frac{X_{L}(i, e, \alpha)}{X_{L}\left(\lambda, 30^{\circ}, 0^{\circ}, 30^{\circ}\right)} \frac{f(\alpha)}{f\left(30^{\circ}\right)}
$$

where, $X_{L}$ is a specific form of a disk function describing the $i$ - and $e$-dependencies at a given $\alpha$, and $f$ is a phase function describing the $\alpha$ dependencies of the surface reflectance. The detailed forms of these functions are provided in the original paper [29]. In addition, the lunar surface radiance $R_{S P}\left[\mathrm{~W} \mathrm{~m}{ }^{-2} \mu \mathrm{m}^{-1} \mathrm{sr}^{-1}\right]$ is calculated as

$$
R_{S P}(\lambda)=r_{\text {sim }}(\lambda, i, e, \alpha) \frac{I_{S u n}(\lambda)}{\pi}\left(\frac{D}{1 \mathrm{AU}}\right)^{2}
$$

where, $I_{\text {Sun }}$ is the solar irradiance $\left(\mathrm{W} \mathrm{m}^{-2} \mu \mathrm{m}^{-1}\right)$ at a distance of $1 \mathrm{AU}$, and $D$ represents the distance between the Sun and the Moon in the astronomical unit (AU]. The positions of the Moon and the satellite were calculated using a two-line element set (TLE) of the satellite (www.space-track.org, accessed on 16 February 2021) and SPICE kernels [32]. The OOC's relative spectral response (RSR) functions (Figure 2) were considered, and the diskintegrated Moon irradiance was predicted for each observation. It should be noted that the SP model covers only $512.6-1600 \mathrm{~nm}$, whereas the ROLO model covers $350-2500 \mathrm{~nm}$; thus, the shorter wavelength bands of OOC-1 and -2 were not compatible with the SP model.

In the case of the SP model, the original output is a radiance map (Figure 4), which was converted to irradiance in the same manner as conducted for the observation image analysis (see Section 2.3). The SP model provides a radiance factor that corresponds to the reflectance standardized with the specified solar incident angle $(i)$, emission angle $(e)$, and phase angle $(\alpha)$ of $30^{\circ}, 0^{\circ}$, and $30^{\circ}$, respectively. The disk-resolved lunar reflectance model has a resolution of $0.5^{\circ} \times 0.5^{\circ}$ in lunar latitude and longitude based on SP hyperspectral data [30]. The SP model can generate the Moon image, with radiance values located at the center point of each pixel. As the effective spatial resolution of the SP model is better than that of OOC, the Moon image was simulated by the SP model with eight times higher 
resolution than OOC in advance. Later it was reduced to the same resolution by the binning process.

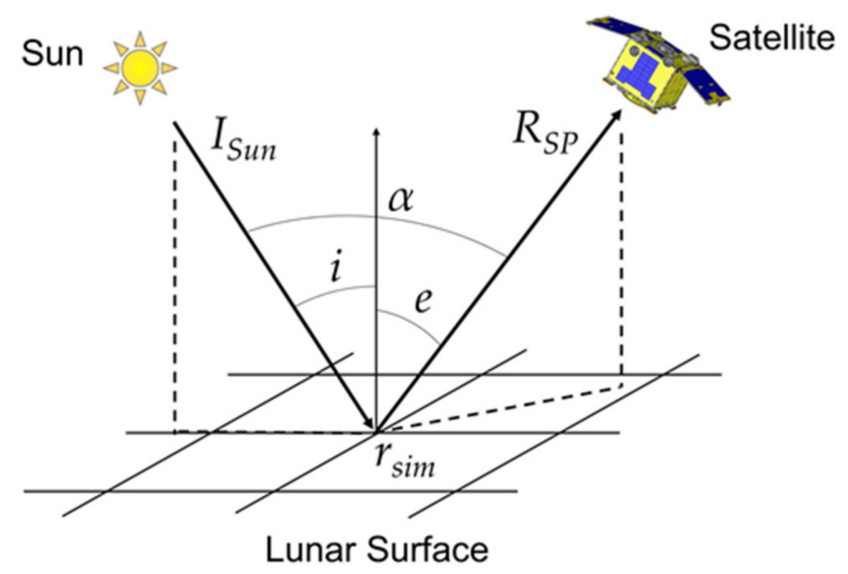

Figure 3. Definition of the solar incident angle (i), emission angle (e), and phase angle $(\alpha)$ used for the simulation of the lunar surface radiance $\left(R_{S P}\right)$ calculated from the solar irradiance $\left(I_{\text {Sun }}\right)$ and the radiance factor $\left(r_{\text {sim }}\right)$ in the Spectrum Profiler $(\mathrm{SP})$ model.
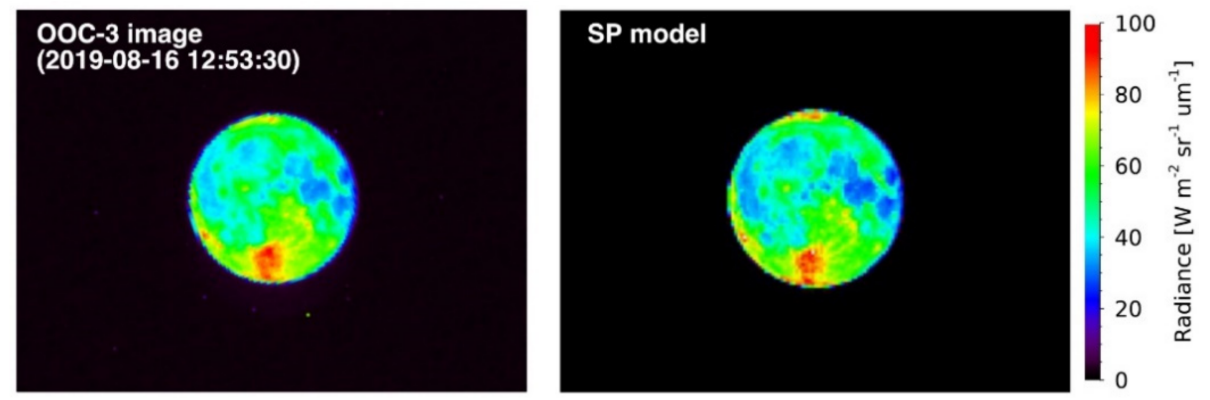

Figure 4. Moon images captured by the OOC $-3(555 \mathrm{~nm})$ band (left) and simulated by the SP model (right). Both images are cropped to $165 \times 124$ pixels, and the observation image is rotated.

\subsection{Radiometric Calibration}

Radiometric correction was performed for all the obtained OOC images using prelaunch calibration data (Figure 5). In advance of the radiometric correction, the observed Moon images were processed by bit conversion, offset reduction, and effective pixel extraction. The corrected radiance of each image pixel was stored as a 16-bit digital number, $\mathrm{DN}=R_{T O A}\left(\mathrm{~W} \mathrm{~m}^{-2} \mathrm{sr}^{-1} \mu \mathrm{m}^{-1}\right) \times 100$, where $R_{T O A}$ is the top-of-atmosphere (TOA) spectral radiance. Additional image correction was conducted to subtract background radiance. Figure 6 depicts the low-illumination enhanced image and processed image. To exclude the background, the center of the Moon in the pixel coordinates $\left(C_{X}, C_{Y}\right)$ was identified by cross-correlation matching with the observation and SP simulated images. Considering the radius of the Moon $L_{M}[\mathrm{~km}]$, satellite-Moon distance $D_{M}[\mathrm{~km}]$, and the instrument instant FOV $\theta_{i}=1.483 \times 10^{-4}(\mathrm{rad} / \mathrm{pixel})$, the Moon disk area was defined as the inner region of Moon radius $L_{P}+2$ pixel, where $L_{P}=\arctan \left(L_{M} / D_{M}\right) / \theta_{i}$. The background radiance was measured in the annular region bounded by $L_{P}+10$ and $L_{P}+20$ circles, and the background was subtracted. In some images, ghost noise appeared in the off-Moon-disk area but may have resulted in a minor effect on the subtraction. 


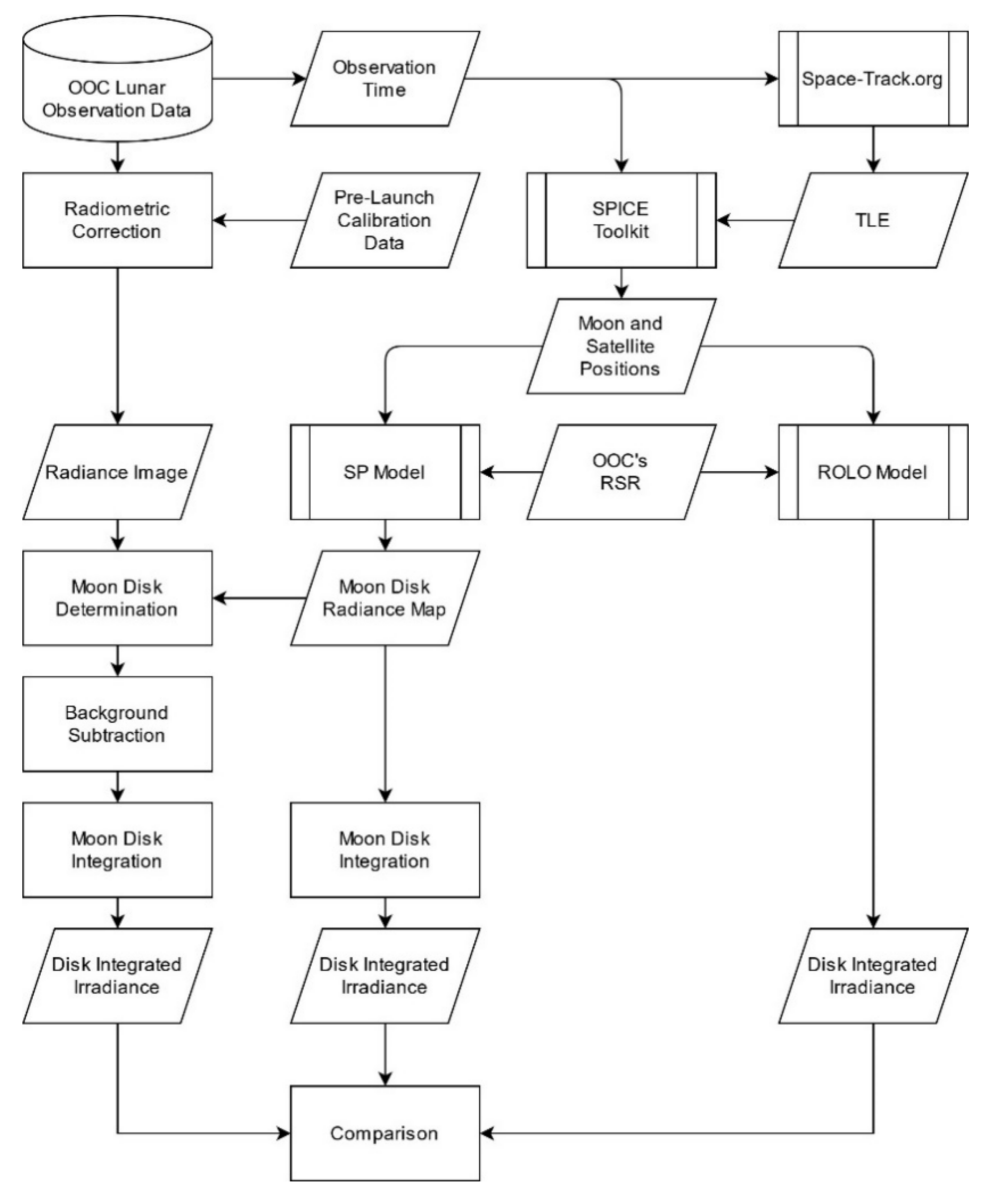

Figure 5. A flow chart of the lunar calibration using pre-launch calibration data.

OOC-3 image

(2019-08-16 12:53:30)

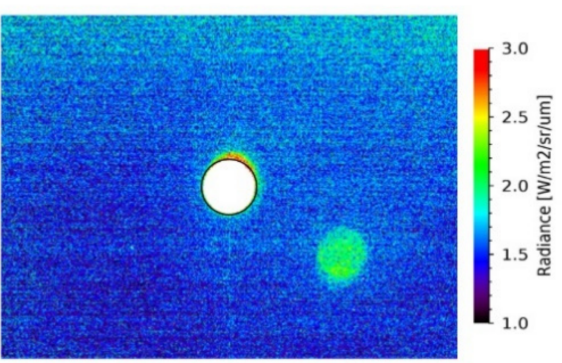

Processed image

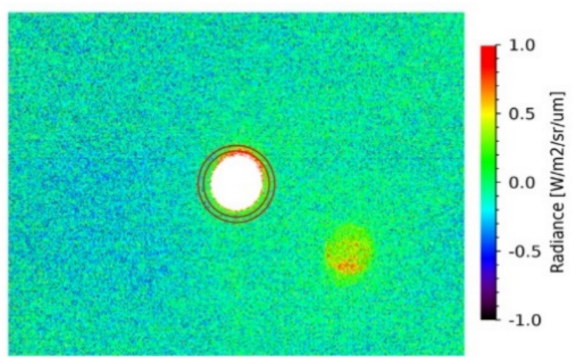

Figure 6. Low-illumination enhanced image of Figure 4 (left) and processed one after the subtraction of background radiance (right). Moon position was centered, and $500 \times 375$ pixels of surrounding background are displayed. Black circle in the left panel indicates the defined Moon limb $\left(L_{P}+2\right.$ pixel $)$. The additional background level was calculated from the red annular region shown in the right panel (see text for detail definitions).

To compare with the irradiance predicted by the ROLO model, we need to integrate the irradiance $\left(\mathrm{W} \mathrm{m}^{-2} \mu_{\mathrm{m}}^{-1}\right.$ ) over the disk both for observation and the SP model-simulated images. The Moon irradiance was calculated as

$$
I=\sum_{i} R_{i} \omega
$$

where, the subscript $i$ indicates the $i$ th pixel including the Moon disk region, $R_{i}$ is the radiance at the $i$ th pixel, and $\omega$ is the instantaneous FOV of the pixels (in the case of OOC, $\left.\omega=\left(1.483 \times 10^{-4}\right)^{2}(\mathrm{str})\right)$. The SP model tends to have a $>5 \%$ error in high incident-angle re- 
gions (near the terminator) and high-emission angle regions (near the limb). Kouyama et al. used the SP model excluding regions with solar incident angle $i>60^{\circ}$ and emission angle $e$ $>45^{\circ}$ from their pixel-based comparison [30]. In this study, we integrated the entire Moon disk pixels because the small diameter of the observed Moon ( $\sim 55$ pixels) causes difficulties in excluding these regions. This point is discussed in Section 4 by comparing the results of the ROLO model.

\section{Results}

\subsection{Sensor Performance Change and Temperature Dependences}

The observed and model-simulated Moon irradiance were compared to investigate the sensor performance and its temporal change. Initially, the degradation of the sensors in space were evaluated. Figure 7 shows the temporal variations in the observationto-simulation irradiance ratio (OSR). As the model could predict the Moon brightness precisely ( $1 \%$ with ROLO), the sensor performance change could be identified as the relative change in the OSR. During 16 months of monitoring from August 2019, OOC-1 depicted an increasing trend in the OSR, while the other bands did not depict clear trends in the OSRs. The OSRs with the ROLO model changed by up to $3 \%$ in each observation month. The SP model irradiance was $\sim 4 \%$ larger and $\sim 1.5 \%$ smaller than the ROLO model irradiance in OOC-3 and OOC-4, respectively, while the deviation of the SP-toROLO irradiance ratio was $<1 \%$. The positive and negative lunar phase angles did not significantly affect OSR variation.

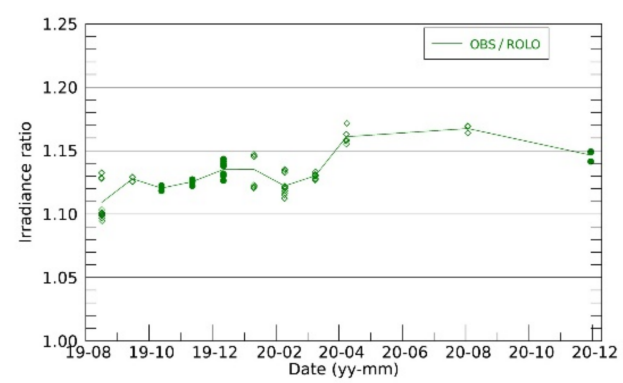

(a) OOC-1 (405 nm)

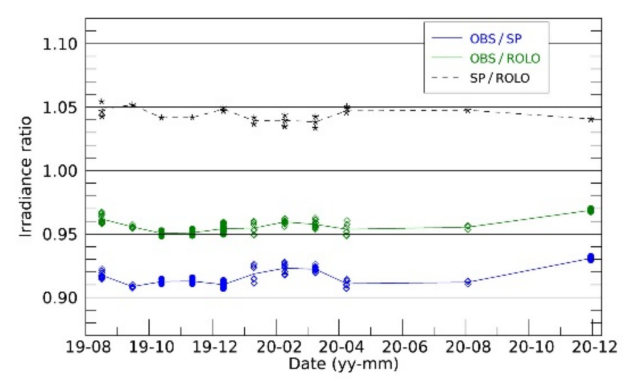

(c) OOC-3 (555 nm)

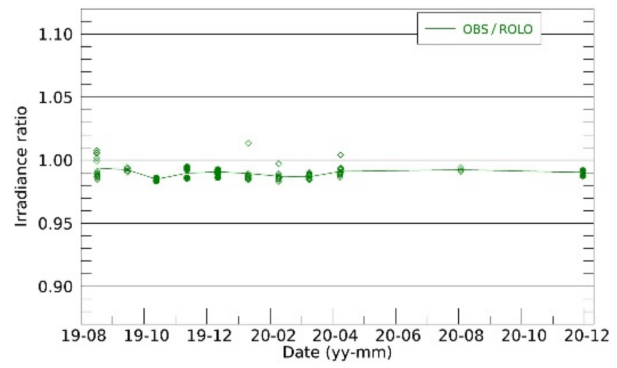

(b) OOC-2 (490 nm)

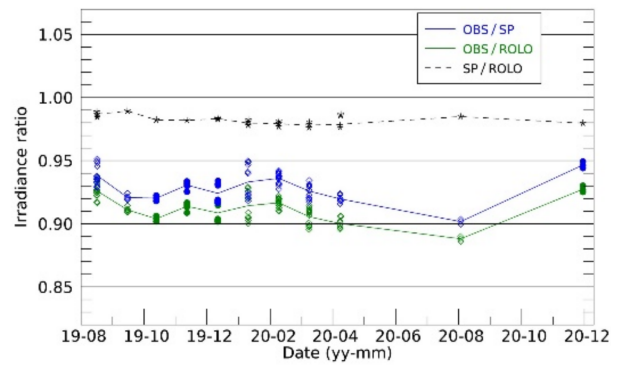

(d) OOC-4 $(869 \mathrm{~nm})$

Figure 7. Temporal changes in the observation-to-simulation irradiance ratio (OSR) without the correction of temperature dependence of each sensor (see main text). The ROLO (green) and SP (blue) based OSR, and SP-to-ROLO irradiance ratio (black) are shown in OOC-3 and -4, where the SP model covers the observation wavelength. Waning Moon and waxing Moon are distinguished with filled circles and open diamonds, respectively.

Our results presented a large variation in the OSRs, implying the existence of other factors affecting the sensor performance. In Figure 8, the sensor temperature was analyzed to determine the potential correlations with the OSRs. Although the OOC measured the sensor temperature not at the CCD image sensor but at the sensor housing (as indicated in Figure 1), it was found that the OSRs were dependent on the sensor temperature. The 
observed negative and positive dependences were major in OOC- 1 and OOC-4, respectively, but they were minor in OOC-2 and OOC-3. Therefore, we performed linear fitting for each scatter and the derived slopes were $-2.2 \times 10^{-3},-1.4 \times 10^{-4}, 5.8 \times 10^{-4}, 1.8 \times 10^{-3}\left(/{ }^{\circ} \mathrm{C}\right)$ for OOC- $1 / 2 / 3 / 4$, respectively.

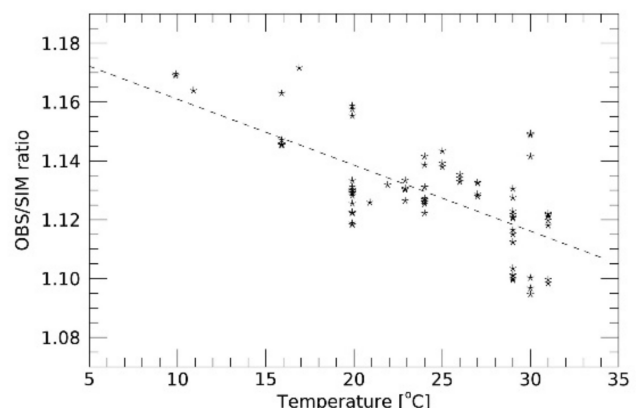

(a) OOC-1 (405 nm)

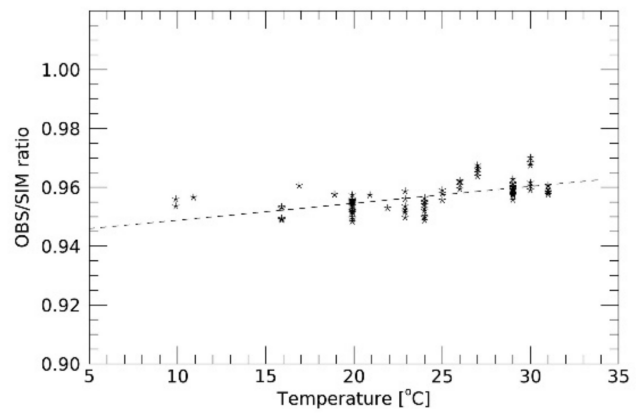

(c) OOC-3 $(555 \mathrm{~nm})$

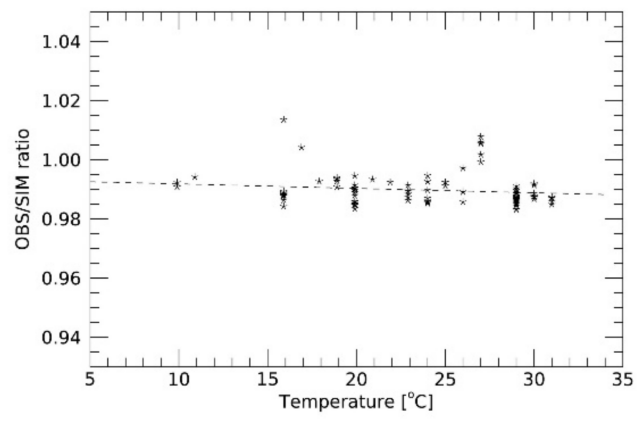

(b) OOC-2 (490 nm)

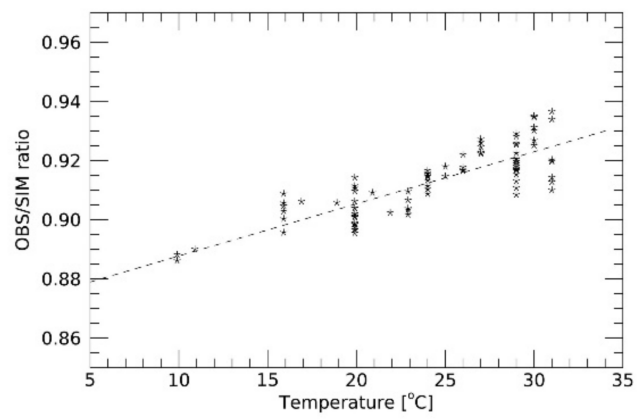

(d) OOC-4 (869 nm)

Figure 8. Observation-to-simulation irradiance ratio (OSR) scatters as a function of the sensor temperature. Dashed lines are the results of the linear fitting.

Based on the linear fitting results, a temperature dependence correction was performed for all the OSRs to be normalized at a reference temperature of $20^{\circ} \mathrm{C}$. Figure 9 shows that the temperature-corrected OSRs do not exhibit obvious sensitivity degradation. OOC-1 still indicates an increasing trend; however, further observations are necessary to conclude this trend.

\subsection{Validation of Lunar Calibration}

A comparison between the observed and simulated irradiance could help to determine the discrepancy between the previous radiometric calibration based on the pre-launch experiment data, and the current lunar calibration. Figure 10 displays the averaged OSR against the ROLO model obtained from all the observations. There is a clear bluing trend in which OOC bands of shorter wavelengths indicate larger irradiance ratios. As the sensor sensitivity degradation cannot be confirmed in this study, the bluing trend might occur due to the launch impact or at the early stage of the satellite operation in space. 


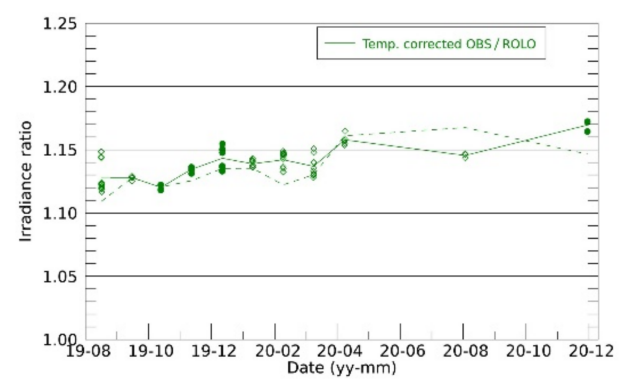

(a) OOC-1 (405 nm)

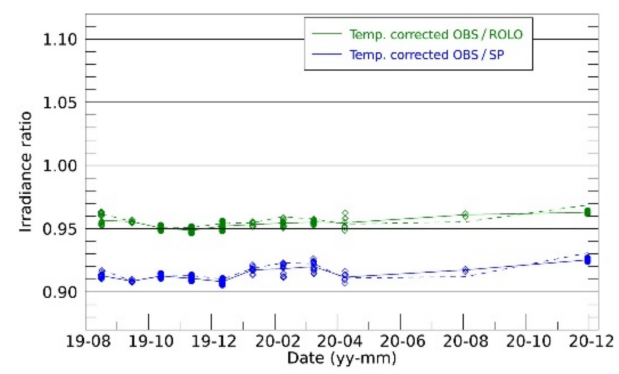

(c) OOC-3 (555 nm)

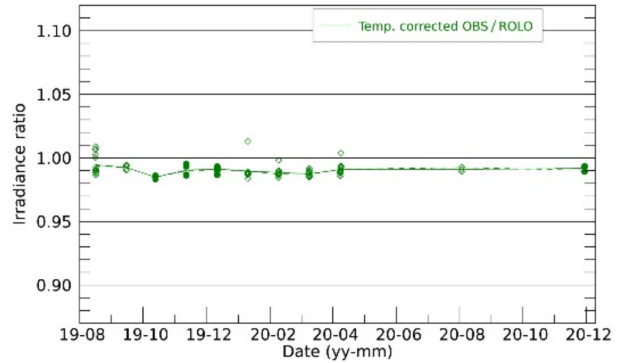

(b) OOC-2 (490 nm)

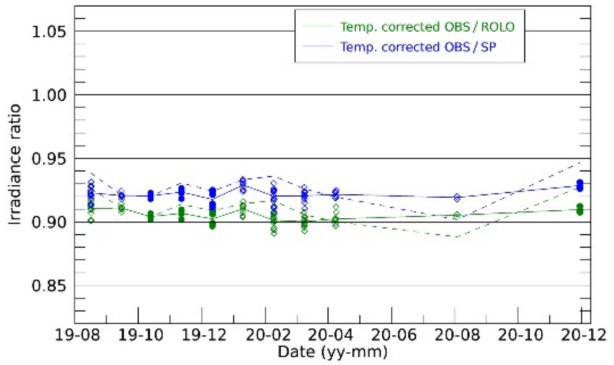

(d) OOC-4 (869 nm)

Figure 9. OSR plots from Figure 7, with corrected temperature dependences. Dashed lines indicate the OSRs before the correction.

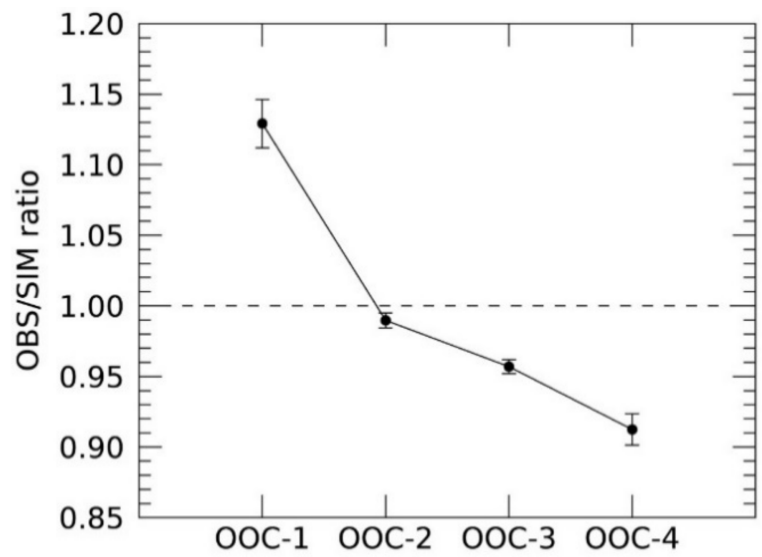

Figure 10. Averaged OSR for each observation band was obtained from all observation. Horizontal dashed line indicates the expected ratio based on the ROLO model, and the error bar indicates the standard deviation, in which the OOC's temperature dependence is included.

To validate this bluing trend, an observation of the vicarious calibration site was conducted. Validation of a result of sensor sensitivity calibration requires to confirm consistency of the result with other methods. One of the most reliable vicarious calibration methods is to observe a well-maintained calibration site in an appropriate condition with a simultaneous ground-based observation and weather monitoring. Five observations were attempted targeting Railroad Valley Playa, which is a vicarious calibration site located $150 \mathrm{~km}$ east of Tonopah, Nevada. Based on the site view image of Railroad Valley [33], an observation date of 20 October 2020 was selected as an appropriate date for validation when there were no clouds or cirrus clouds above the site. The radiometric calibration network (RadCalNet) TOA reflectance data, developed by a working group of the Committee on Earth Observation Satellites Working Group on Calibration and Validation (CEOSWGCV) [34], was utilized. The radiometric calibration test site (RadCaTS) in Railroad 
Valley has a square area of $1 \mathrm{~km} \times 1 \mathrm{~km}$ centered at a longitude of $115.690^{\circ} \mathrm{W}$ and latitude of $38.497^{\circ} \mathrm{N}$. The OOC observed this area at 16:36:31.500 UTC with $10 \times 10$ pixels, when the local solar time was 09:09 and the viewing angle was $24.62^{\circ}$. The closest RadCaTS TOA reflectance data were recorded at 17:00 UTC; thus, there was a difference of $\sim 30 \mathrm{~min}$ between the datasets. The TOA radiance of OOC $\left(R_{T O A}\right)$ was converted to the TOA reflectance as

$$
\rho=\pi R_{T O A} d^{2} / I \cos \Phi
$$

where, $\rho$ is the TOA reflectance, $d$ is the Earth-Sun distance in astronomical units, $I$ is the solar irradiance, and $\Phi$ is the solar zenith angle. The Chance/Kurucz solar irradiance spectral model from MODTLAN V5.2 was utilized, as it was used to predict TOA reflectance in RadCalNet [35-37]. The solar irradiance was calculated from the numerical convolution of the OOC's RSR function with solar spectral irradiance, which is a merged spectrum of $[38,39]$.

Figure 11 shows a comparison of TOA reflectance between the RadCaTS and OOC observations. After applying the lunar calibration result derived from Figure 10, the bluing trend recovered significantly and was consistent with the RadCalNet reflectance in the visible range. Although the observation time of the OOC was 30 min earlier than that of RadCalNet in the morning, the OOC's TOA reflectance was slightly higher than that of RadCalNet. This might be attributed to the OOC observation viewing angle of $24.62^{\circ}$. Here, the uncertainties of the bidirectional reflectance distribution function effect and atmospheric correction was not considered. Although the lunar calibration provided reasonable corrections for OOC- $1 / 2 / 3$, the deviation in the OOC- 4 was increased by lunar calibration. This deviation is discussed in detail in the following section.

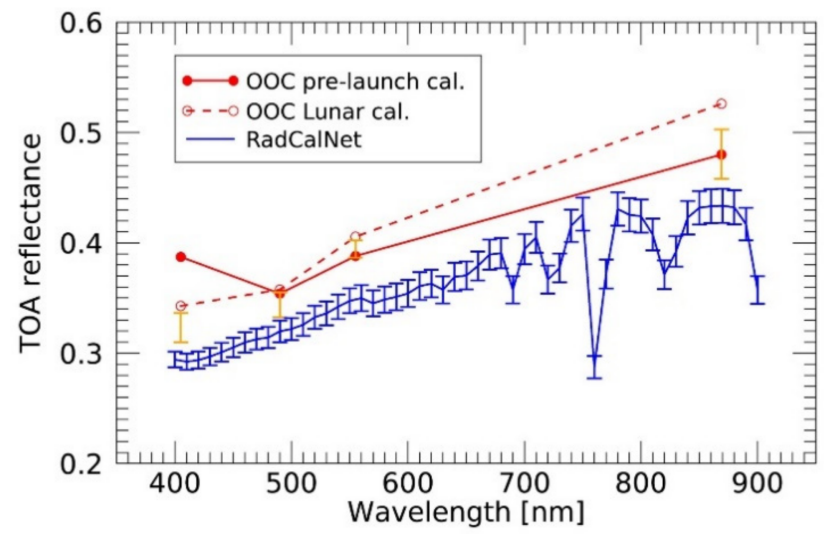

Figure 11. Top-of-atmosphere (TOA) reflectance spectrum of RadCaTS (blue) from RadCalNet and OOC observation (red). Both pre-launch calibration (solid line) and the current lunar calibration result (dashed line) are plotted, and the orange error bar indicates the potential spectrum change due to the superposed background illumination (see Section 4).

\section{Discussion}

In this study, the two Moon models of ROLO and SP were utilized. The results of both models indicated that the OOC has no significant sensor sensitivity degradation. However, the SP model irradiance was $\sim 4 \%$ larger and $\sim 1.5 \%$ smaller than the ROLOs in OOC-3 and OOC-4, respectively. The original SP model has $\sim 10 \%$ uncertainty in the absolute radiance, and there are correction coefficients to modify the SP radiance to be consistent with the ROLO's integrated irradiance. The correction coefficients are a function of the wavelength, and the coefficients estimated by the previous work at a negative phase angle of $-27.7^{\circ}$ were used (see Section 3.2 of [30]). As our Moon observations were conducted in a rather small phase angle of $5.5^{\circ}-12.5^{\circ}$, the discrepancy between the ROLO and SP model irradiance in OOC-3 and 4 was attributed mainly to the phase angle difference. Another possible explanation for this discrepancy is the relatively large uncertainty of the SP model radiance in the high-incident-angle region near the terminator of the Moon, 
and in the high-emission angle region near the limb of the Moon. Although the SP model has the advantage of providing disk-resolved Moon radiance, pixel-based comparisons were recommended solely in the regions where solar incident angle was less than $60^{\circ}$ and emission angle was less than $45^{\circ}$, to avoid unexpected radiance bias [30]. However, the small discrepancy between the ROLO and SP models indicates that the SP model can be utilized to calculate the relative irradiance change even if the observed Moon image is small with a diameter of $\sim 50$ pixels.

Sensor sensitivity dependence on temperature is one of the main topics of this study. Although the OOC did not measure the sensor temperature precisely for each component, clear sensitivity dependencies on the sensor temperature were formed. The sensor temperature can be changed owing to the solar illumination conditions of the satellite in orbit and internal heating from the electric circuits. One of the notable results from this study is that the sensitivity dependence on temperature varies significantly with wavelength; a negative correlation was observed in OOC- 1 at $405 \mathrm{~nm}$, but positive in OOC-4 at $869 \mathrm{~nm}$. Recently, a similar relationship was reported in optical navigation cameras (ONCs) onboard Hayabusa-2, which is a Japanese sample return mission from the asteroid's surface (see Section 3.9 .2 of [40]). Both studies indicate that shorter (longer) observation wavelengths have a negative (positive) correlation with temperature. In the OOC, the relationship is reversed at wavelengths ranging from 495 to $555 \mathrm{~nm}$, but it was approximately $800 \mathrm{~nm}$ in the ONCs. Thermal analysis of the optical sensor system is required to elucidate this relationship. Although further interpretation is beyond the scope of this study, our results emphasize the importance of measuring the sensor temperature, especially for optical sensors in which commercial off-the-shelf components are used.

After the correction of temperature dependencies, no significant sensitivity degradation was confirmed during the 16 months of the Moon observation period for half a year after the launch. However, a comparison of the observation and the ROLO's simulation irradiance revealed a prominent bluing trend in OOC's sensor sensitivity. This bluing trend was validated by comparing the TOA reflectance spectrum of the Railroad Valley Playa from RadCalNet. Although the derived re-calibration parameter is consistent for OOC- $1 / 2 / 3$, we could not have a conclusive interpretation of the deviation in OOC-4. One possibility is that the commercial lens used for OOC cameras is designed suitable for imaging in visible region, and thus OOC-4 band (infrared) is out of the design and may be contaminated by stray light in infrared region. Figure 12 depicts the background irradiance level, which can be caused by the contamination of stray light, to be subtracted from the Moon irradiance in the calibration process. From the analysis of Moon observations, the OOC-1/2/3/4 images have background contamination equal to $\sim 4 \%, \sim 3 \%, \sim 2.5 \%$, and $\sim 8 \%$ of the Moon irradiance on average, respectively. The background contamination in OOC-4 is more than twice as large as that in the other bands; thus, similar background contamination will be included in the Earth observation images. In Figure 11, the possible reflectance spectrum is shown with orange error bars, assuming that background contamination was included in the images of Railroad Valley Playa. The minimum and maximum background-to-observation irradiance ratios were used to estimate the error bar range. Although the most appropriate cloud condition was selected from the five observations, the observation time and the viewing angle were not optimum for the validation. The different observation time may cause a disparity in atmospheric condition and the off-nadir viewing angle can contribute to bidirectional reflectance distribution function effects. Further vicarious observations are necessary to validate OOC's bluing trend and to investigate the contamination in the Earth observation images, while the current results indicate that lunar observation could help to estimate the contamination of stray light in the Earth observation image. 


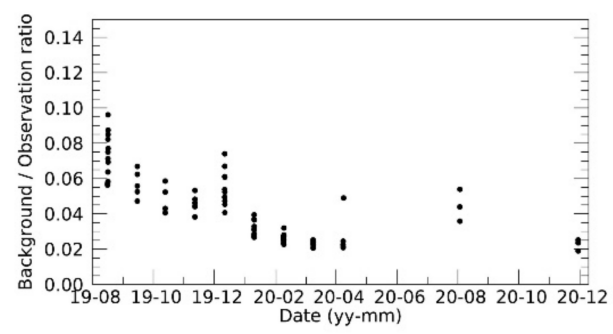

(a) OOC-1 (405 nm)

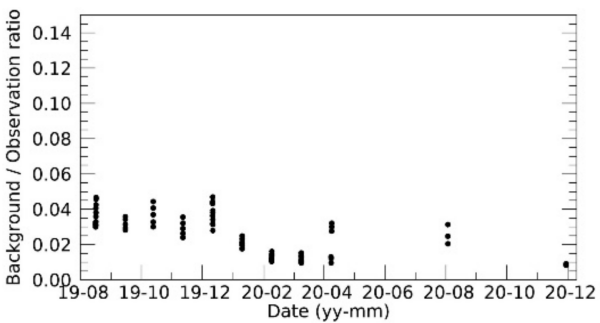

(c) OOC-3 $(555 \mathrm{~nm})$

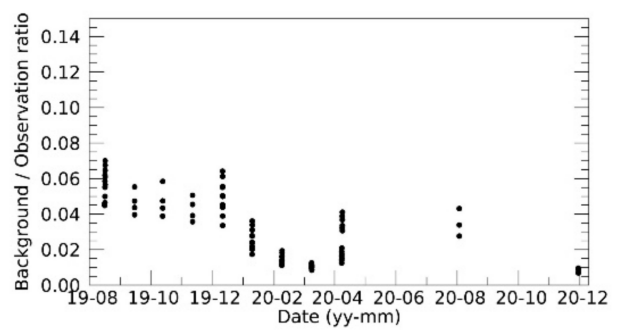

(b) OOC-2 (490 nm)

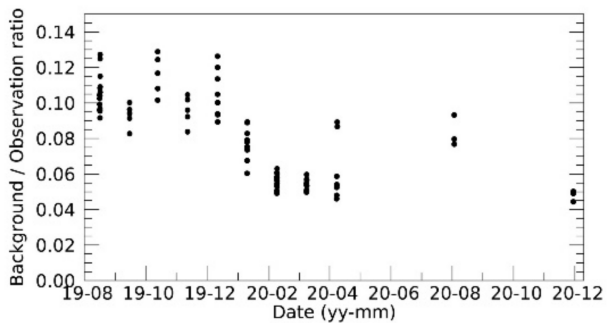

(d) OOC-4 $(869 \mathrm{~nm})$

Figure 12. Temporal changes in the background-to-observation irradiance ratio.

\section{Conclusions}

Radiometric calibration using the ROLO and SP Moon models in space (lunar calibration) was conducted for a microsatellite named RISESAT launched in 2019 and its multispectral camera OOC. The OSR derived from both the ROLO and SP models can be utilized to reveal the dependence of the sensor sensitivity on the instrument temperature. After correcting the temperature dependency, no significant sensitivity degradation was observed in the OOC. Despite the small temporal change in sensitivity, there is a non-negligible discrepancy in the OOC's sensor sensitivity, where a shorter observation wavelength shows larger irradiance against the ROLO model irradiance. To validate this bluing trend, vicarious observations targeting Railroad Valley Playa were conducted. By comparing the TOA reflectance obtained from RadCalNet, it was found that the derived parameter of the lunar calibration was tentatively valid for correcting the current OOC's bluing trend in the visible range. Although the lunar calibration parameter for the infrared band was unexpectedly inconsistent with vicarious calibration, stray light contamination can be a plausible explanation, and lunar calibration could potentially contribute to estimation of the contaminated background irradiance in the Earth observation images.

Radiometric calibration with the Moon does not require any special equipment as long as the satellite satisfies the thermal balance and attitude control requirements for Moon observation. Therefore, lunar calibration can be a useful radiometric calibration method for optical sensors on nano/microsatellites, which have payload and cost restrictions. The relative irradiance derived from the SP model is consistent with that of the ROLO model with $\sim 1 \%$ accuracy. As the SP model can provide a spatially resolved Moon radiance map, the SP model must be used for calibrating high-spatial-resolution optical sensors whose FOV is not wide enough to capture the full Moon disk.

Author Contributions: Writing—original draft preparation, M.I. and J.K.; investigation and formal analysis, M.I.; data curation, J.K.; methodology, software, and supervision, T.K. (Toru Kouyama); investigation and resources, T.K. (Toshinori Kuwahara), S.F., Y.S. (Yuji Sakamoto), and Y.S. (Yuji Sato); validation, H.Y.; funding acquisition, J.K., T.K. (Toru Kouyama), S.-I.S., T.H., and Y.T. All authors have read and agreed to the published version of the manuscript.

Funding: This research and development work was supported by the MIC/SCOPE \#191502001. This study was also partly supported by JSPS KAKENHI Grant Number 19K14789.

Institutional Review Board Statement: Not applicable. 
Informed Consent Statement: Not applicable.

Data Availability Statement: The data presented in this study are available on request from the corresponding author.

Acknowledgments: The authors would like to thank Naoya Shiraishi and Kazuki Kibune, Department of Aerospace Engineering, Tohoku University, for supporting the satellite operation.

Conflicts of Interest: The authors declare no conflict of interest.

\section{References}

1. Denis, G.; Claverie, A.; Pasco, X.; Darnis, J.-P.; de Maupeou, B.; Lafaye, M.; Morel, E. Towards Disruptions in Earth Observation? New Earth Observation Systems and Markets Evolution: Possible Scenarios and Impacts. Acta Astronaut. 2017, 137, 415-433. [CrossRef]

2. Li, J.; Roy, D.P. A Global Analysis of Sentinel-2A, Sentinel-2B and Landsat-8 Data Revisit Intervals and Implications for Terrestrial Monitoring. Remote Sens. 2017, 9, 902. [CrossRef]

3. Francini, S.; McRoberts, R.E.; Giannetti, F.; Mencucci, M.; Marchetti, M.; Mugnozza, G.S.; Chirici, G. Near-Real Time Forest Change Detection Using PlanetScope Imagery. Eur. J. Remote Sens. 2020, 53, 233-244. [CrossRef]

4. Niroumand-Jadidi, M.; Bovolo, F.; Bruzzone, L.; Gege, P. Physics-Based Bathymetry and Water Quality Retrieval Using PlanetScope Imagery: Impacts of 2020 COVID-19 Lockdown and 2019 Extreme Flood in the Venice Lagoon. Remote Sens. 2020, 12, 2381. [CrossRef]

5. $\quad$ Park, S.-H.; Jung, H.-S.; Lee, M.-J.; Lee, W.-J.; Choi, M.-J. Oil Spill Detection from PlanetScope Satellite Image: Application to Oil Spill Accident near Ras Al Zour Area, Kuwait in August 2017. J. Coast. Res. 2019, 90, 251-260. [CrossRef]

6. Houborg, R.; McCabe, M.F. High-Resolution NDVI from Planet's Constellation of Earth Observing Nano-Satellites: A New Data Source for Precision Agriculture. Remote Sens. 2016, 8, 768. [CrossRef]

7. Leach, N.; Coops, N.C.; Obrknezev, N. Normalization Method for Multi-Sensor High Spatial and Temporal Resolution Satellite Imagery with Radiometric Inconsistencies. Comput. Electron. Agric. 2019, 164, 104893. [CrossRef]

8. Houborg, R.; McCabe, M.F. A Cubesat Enabled Spatio-Temporal Enhancement Method (CESTEM) Utilizing Planet, Landsat and MODIS Data. Remote Sens. Environ. 2018, 209, 211-226. [CrossRef]

9. Houborg, R.; McCabe, M.F. Daily Retrieval of NDVI and LAI at $3 \mathrm{~m}$ Resolution via the Fusion of CubeSat, Landsat, and MODIS Data. Remote Sens. 2018, 10, 890. [CrossRef]

10. Latte, N.; Lejeune, P. PlanetScope Radiometric Normalization and Sentinel-2 Super-Resolution (2.5 m): A Straightforward Spectral-Spatial Fusion of Multi-Satellite Multi-Sensor Images Using Residual Convolutional Neural Networks. Remote Sens. 2020, 12, 2366. [CrossRef]

11. Markham, B.; Barsi, J.; Kvaran, G.; Ong, L.; Kaita, E.; Biggar, S.; Czapla-Myers, J.; Mishra, N.; Helder, D. Landsat-8 Operational Land Imager Radiometric Calibration and Stability. Remote Sens. 2014, 6, 12275-12308. [CrossRef]

12. Kabir, S.; Leigh, L.; Helder, D. Vicarious Methodologies to Assess and Improve the Quality of the Optical Remote Sensing Images: A Critical Review. Remote Sens. 2020, 12, 4029. [CrossRef]

13. Kieffer, H.H. Photometric Stability of the Lunar Surface. Icarus 1997, 130, 323-327. [CrossRef]

14. Jumpasut, A.; Fukuzato, A.; Greenberg, J.; Wilson, N. Lunar Radiometric Calibration on Planet Dove Satellites. In Proceedings of the Conference on Characterization and Radiometric Calibration for Remote Sensing (CALCON), Logan, UT, USA, 21-24 August 2017.

15. Jumpasut, A.; Fukuzato, A.; Zuleta, I. Using the Moon as a Calibration Source for a Fleet of Satellites. In Proceedings of the Conference on Characterization and Radiometric Calibration for Remote Sensing (CALCON), Logan, UT, USA, 18-21 June 2018.

16. Kouyama, T.; Nakamura, R.; Kato, S.; Miyashita, N. One-Year Lunar Calibration Result of Hodoyoshi-1, Moon as an Ideal Target for Small Satellite Radiometric Calibration. In Proceedings of the Small Satellite Conference, Logan, UT, USA, 4-9 August 2018.

17. Jiang, Y.; Shi, Y.; Li, L.; Xu, M.; Zeng, W.; Jiang, Y.; Li, Z. On-Orbit Radiance Calibration of Nighttime Sensor of LuoJia1-01 Satellite Based on Lunar Observations. Remote Sens. 2019, 11, 2183. [CrossRef]

18. Kuwahara, T.; Yoshida, K.; Sakamoto, Y.; Takahashi, Y.; Kurihara, J.; Yamakawa, H.; Takada, A. A Japanese microsatellite bus system for international scientific missions. In Proceedings of the 62nd International Astronautical Congress, IAC 2011, Cape Town, South Africa, 3-7 October 2011; pp. 3699-3706.

19. Kuwahara, T.; Yoshida, K.; Sakamoto, Y.; Tomioka, Y.; Fukuda, K.; Fukuyama, M.; Shibuya, Y. International Scientific MicroSatellite RISESAT Based on Space Plug and Play Avionics. In Proceedings of the Small Satellite Conference, Logan, UT, USA, 13-16 August 2012.

20. Morel, A. Minimum Requirements for an Operational Ocean-Colour Sensor for the Open Ocean; Reports of the International Ocean Colour Coordinating Group; IOCCG: Dartmouth, NS, Canada, 1998; p. 1.

21. Sugimura, N.; Kuwahara, T.; Yoshida, K. Attitude Determination and Control System for Nadir Pointing Using Magnetorquer and Magnetometer. In Proceedings of the 2016 IEEE Aerospace Conference, Big Sky, MT, USA, 5-12 March 2016 ; pp. 1-12. 
22. Fujita, S.; Sato, Y.; Kuwahara, T.; Sakamoto, Y.; Yoshida, K. On-ground verification of attitude control system for 50-kg-class microsatellite using a hardware-in-the-loop-simulator. In Proceedings of the 68th International Astronautical Congress, IAC 2017, Adelaide, SA, Australia, 25-29 September 2017; pp. 6141-6150.

23. Fujita, S.; Sato, Y.; Kuwahara, T.; Sakamoto, Y.; Yoshida, K. Attitude Maneuvering Sequence Design of High-Precision Ground Target Tracking Control for Multispectral Earth Observations. In Proceedings of the 2019 IEEE/SICE International Symposium on System Integration (SII), Paris, France, 14-16 January 2019; pp. 153-158.

24. Kaydash, V.; Pieters, C.; Shkuratov, Y.; Korokhin, V. Lunar Opposition Effect as Inferred from Chandrayaan-1 M3 Data. J. Geophys. Res. Planets 2013, 118, 1221-1232. [CrossRef]

25. Kieffer, H.H.; Stone, T.C. The Spectral Irradiance of the Moon. Astron. J. 2005, 129, 2887. [CrossRef]

26. Stone, T.C. Radiometric Calibration Stability and Inter-Calibration of Solar-Band Instruments in Orbit Using the Moon. In Proceedings of the Earth Observing Systems XIII, International Society for Optics and Photonics, San Diego, CA, USA, 11-13 August 2008; Volume 7081, p. 70810X.

27. Choi, T.; Shao, X.; Cao, C. On-Orbit Radiometric Calibration of Suomi NPP VIIRS Reflective Solar Bands Using the Moon and Solar Diffuser. Appl. Opt. 2018, 57, 9533-9542. [CrossRef]

28. Cao, C.; Zhang, B.; Shao, X.; Wang, W.; Uprety, S.; Choi, T.; Blonski, S.; Gu, Y.; Bai, Y.; Lin, L.; et al. Mission-Long Recalibrated Science Quality Suomi NPP VIIRS Radiometric Dataset Using Advanced Algorithms for Time Series Studies. Remote Sens. 2021, 13, 1075. [CrossRef]

29. Yokota, Y.; Matsunaga, T.; Ohtake, M.; Haruyama, J.; Nakamura, R.; Yamamoto, S.; Ogawa, Y.; Morota, T.; Honda, C.; Saiki, K.; et al. Lunar Photometric Properties at Wavelengths 0.5-1.6 $\mu \mathrm{m}$ Acquired by SELENE Spectral Profiler and Their Dependency on Local Albedo and Latitudinal Zones. Icarus 2011, 215, 639-660. [CrossRef]

30. Kouyama, T.; Yokota, Y.; Ishihara, Y.; Nakamura, R.; Yamamoto, S.; Matsunaga, T. Development of an Application Scheme for the SELENE/SP Lunar Reflectance Model for Radiometric Calibration of Hyperspectral and Multispectral Sensors. Planet. Space Sci. 2016, 124, 76-83. [CrossRef]

31. Kouyama, T.; Nakamura, R.; Kato, S.; Kimura, M. Moon Observations for Small Satellite Radiometric Calibration. In Proceedings of the 2017 IEEE International Geoscience and Remote Sensing Symposium (IGARSS), Fort Worth, TX, USA, 23-28 July 2017; pp. 3529-3532.

32. Acton, C.; Bachman, N.; Semenov, B.; Wright, E. A Look towards the Future in the Handling of Space Science Mission Geometry. Planet. Space Sci. 2018, 150, 9-12. [CrossRef]

33. Czapla-Myers, J.S.; Thome, K.J.; Leisso, N.P. Radiometric Calibration of Earth-Observing Sensors Using an Automated Test Site at Railroad Valley, Nevada. Can. J. Remote Sens. 2010, 36, 474-487. [CrossRef]

34. Bouvet, M.; Thome, K.; Berthelot, B.; Bialek, A.; Czapla-Myers, J.; Fox, N.P.; Goryl, P.; Henry, P.; Ma, L.; Marcq, S.; et al. RadCalNet: A Radiometric Calibration Network for Earth Observing Imagers Operating in the Visible to Shortwave Infrared Spectral Range. Remote Sens. 2019, 11, 2401. [CrossRef]

35. Berk, A.; Anderson, G.P.; Acharya, P.K.; Shettle, E.P. Modtran 5.2.1 User's Manual; Air Force Research Laboratory, Hanscom AFB: Bedford, MA, USA, 2011.

36. Chance, K.V.; Spurr, R.J.D. Ring Effect Studies: Rayleigh Scattering, Including Molecular Parameters for Rotational Raman Scattering, and the Fraunhofer Spectrum. Appl. Opt. 1997, 36, 5224-5230. [CrossRef]

37. Anderson, G.P.; Picard, R.H.; Chetwynd, J.H. Proceedings of the 17th Annual Conference on Atmospheric Transmission Models, Hanscom AFB, MA, USA, 8-9 June 1994; Phillips Laboratory Research Library-Air Force Phillips Laboratory: Hanscom Air Force Base, MA, USA, 1995.

38. Thuillier, G.; Hersé, M.; Labs, D.; Foujols, T.; Peetermans, W.; Gillotay, D.; Simon, P.C.; Mandel, H. The Solar Spectral Irradiance from 200 to $2400 \mathrm{Nm}$ as Measured by the SOLSPEC Spectrometer from the Atlas and Eureca Missions. Sol. Phys. 2003, $214,1-22$. [CrossRef]

39. Chance, K.; Kurucz, R.L. An Improved High-Resolution Solar Reference Spectrum for Earth's Atmosphere Measurements in the Ultraviolet, Visible, and near Infrared. J. Quant. Spectrosc. Radiat. Transf. 2010, 111, 1289-1295. [CrossRef]

40. Tatsumi, E.; Kouyama, T.; Suzuki, H.; Yamada, M.; Sakatani, N.; Kameda, S.; Yokota, Y.; Honda, R.; Morota, T.; Moroi, K.; et al. Updated Inflight Calibration of Hayabusa2's Optical Navigation Camera (ONC) for Scientific Observations during the Cruise Phase. Icarus 2019, 325, 153-195. [CrossRef] 